\title{
Exact and efficient signal reconstruction in frequency-domain optical-coherence tomography
}

\author{
Chandra Sekhar Seelamantula, ${ }^{1, *}$ Martin L. Villiger, ${ }^{2}$ Rainer A. Leitgeb, ${ }^{3}$ and Michael Unser ${ }^{1}$ \\ ${ }^{1}$ Laboratoire d'imagerie biomédicale, École polytechnique fédérale de Lausanne (EPFL), Switzerland \\ ${ }^{2}$ Laboratoire d'optique biomédicale, École polytechnique fédérale de Lausanne (EPFL), Switzerland \\ ${ }^{3}$ Center for Biomedical Engineering and Physics, Medical University of Vienna, Austria \\ *Corresponding author: chandrasekhar.seelamantula@epfl.ch
}

Received November 9, 2007; accepted March 20, 2008;

posted April 30, 2008 (Doc. ID 89529); published June 26, 2008

\begin{abstract}
We address the problem of tomogram reconstruction in frequency-domain optical-coherence tomography. We propose a new technique for suppressing the autocorrelation artifacts that are commonly encountered with the conventional Fourier-transform-based approach. The technique is based on the assumptions that the scattering function is causal and that the intensity of the light reflected from the object is smaller than that of the reference. The technique is noniterative, nonlinear, and yields an exact solution in the absence of noise. Results on synthesized data and experimental measurements show that the technique offers superior quality reconstruction and is computationally more efficient than the iterative technique reported in the literature. (C) 2008 Optical Society of America
\end{abstract}

OCIS codes: $110.4500,170.3880,110.6955,180.1655$.

\section{INTRODUCTION}

Optical-coherence tomography (OCT) is an efficient interferometric imaging modality that is well-suited for noninvasive three-dimensional imaging of biological specimens. Typically, one can achieve a penetration depth in tissue of up to several millimeters, with a micrometer-range axial resolution. Primarily, there are two types of OCT depending on the mechanism by which the optical signal is detected: (i) time-domain OCT (TDOCT) and (ii) frequencydomain OCT (FDOCT). To acquire the depth information, TDOCT requires a scanner (mechanical displacement of a reference arm) whereas FDOCT can acquire the same information in a single exposure without scanning [1]. This advantage comes from the fact that the inverse Fourier transform of the spectral interference pattern contains the information about the axial sample structure (the inverse scattering theorem) [1-3]. The primary medical applications of FDOCT are tissue imaging, dermatology, and ophthalmology [4-6]. The first medical images for measuring intraocular distances [1] were obtained in 1995 and the first in vivo FDOCT measurements of the human retina were reported in 2002 [7].

FDOCT has some weaknesses that limit its performance. The conventional Fourier transform approach yields the zero-order term of the source, the zero-order term of the sample-also known as the autocorrelation noise, and a complex-conjugate ambiguity term of the object. The complex-conjugate ambiguity can be eliminated by placing the zero-delay plane outside the sample or by using phase-shifting techniques [8-13]. The zero-order term of the source can be suppressed by subtracting the measured source spectrum. The artifacts that cannot be suppressed by the Fourier transform approach are due to the autocorrelation. The artifacts reduce the signal-tonoise ratio (SNR) and resolution. They are also disturbing because they are superimposed on the structure of interest, and may lead to an erroneous interpretation of the specimen morphology. For example, in retinal imaging the artifacts arise due to the strong correlation between the waves reflected from the inner limiting membrane and the retinal pigment epithelium and appear as new morphological structures within the area corresponding to the vitreous of the eye. The artifacts can be kept below the shot noise level by optimally selecting the optical power and exposure time [14]. However, the price to pay for this type of strategy is a decrease in the overall SNRthe effective optical energy being well below the acceptable safety threshold.

Alternatively, the artifacts can be suppressed by employing sophisticated reconstruction techniques. For example, the inverse problem can be reformulated within the framework of phase retrieval and an approximate solution can be computed by employing conventional Fienup or Gerchberg-Saxton iterative algorithms $[15,16]$. The signal can also be modeled as the impulse response of a rational minimum-phase transfer function with constraints on the poles and zeros. The reconstruction problem is then equivalent to one of linear system identification for which iterative [17] or analytic solutions [18] are available. Ozcan et al. [19] have proposed an iterative technique based on a minimum-phase assumption-their reconstruction technique is similar to that of Fienup [15], Bauschke [16], and Quatieri and Oppenheim [17].

In this paper, we exploit an important property of the FDOCT measurements that enables exact reconstruction. We do not assume a rational Fourier transform model but require that the zero-phase delay plane should lie outside the sample-this is indeed the case in many standard FDOCT systems. Our technique is noniterative and hence better suited for real-time imaging applications. 
The organization of the paper is as follows. In Section 2 , we describe the acquisition of the FDOCT signal and also specify the signal model. Section 3 contains a characterization of the artifacts in the conventional Fourierbased reconstruction. In Section 4, we present the new technique for exact reconstruction; the connections to homomorphic signal processing $[20,21]$ are established in Section 5. Sampling issues and implementation details are given in Section 6. In Section 7, we demonstrate the performance of our technique on synthesized as well as experimental data and compare it with the other techniques reported in the literature.

\section{FDOCT SIGNAL ACQUISITION AND MODEL}

In Fig. 1 we show a standard Michelson interferometric setup for making FDOCT measurements. The output of a broadband light source is split into two beams that are directed towards the two arms of an interferometer: (i) the reference arm, which contains a broadband mirror that reflects light and creates the reference signal, and (ii) the object arm, which reaches the specimen. The light that is reflected from scatterers within the specimen contributes to the measured signal. The reference and object-arm signals are coupled into a single-mode fiber and directed towards a spectrometer to obtain a spectral decomposition as a function of the wavelength $\lambda$. The depth information of the object is encoded as a spectral fringe pattern. Increasing the source bandwidth has the effect of increasing the axial resolution, provided that the bandwidth of the spectrometer is not exceeded. The accessible depth range within the specimen is also inversely proportional to the spectrometer resolution. For three-dimensional imaging it is necessary to scan the sample laterally.

Let $a(z)$ denote the amplitude of the light field generated due to scattering by the object, as a function of depth $z$. The spectrometer measurements are mapped onto the wavenumber $k=2 \pi / \lambda$, resulting in the signal

$$
I(k)=S(k)\left|a_{R} e^{j 2 k r}+\int_{-\infty}^{+\infty} a(z) e^{j 2 k[r+n(z) z]} \mathrm{d} z\right|^{2} .
$$

We have used the same notations as those used in [[22], Chapter 12], namely, $2 r$ is the path length in the reference arm; $2 z$ is the path length in the object arm relative

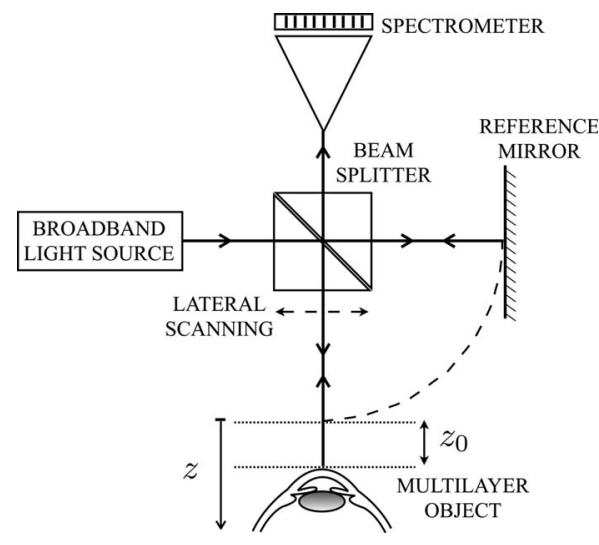

Fig. 1. Schematic of the FDOCT setup. to the reference plane; $n(z)$ is the refractive index as a function of depth in the sample; $a_{R}$ is the amplitude of the wave reflected by the mirror (without loss of generality, we set $\left.a_{R}=1\right) ; a(z)$ is the amplitude of the backscattered wave; and $S(k)$ is the power spectrum of the light source.

$S(k)$ can be measured by completely blocking the object arm; indeed when $a(z)=0$, we have that $I(k)=S(k)$. From Eq. (1), note that we have access to the optical pathlength differences between the reference and object arms, which is what we are interested in. The refractive index $n(z)$ is generally not known a priori; a standard simplification is achieved by making a zeroth-order approximation: $n(z)=n$; i.e., a constant refractive index. This assumption is widely used and holds if the light sources have moderate bandwidth. Further, substituting $\omega$ $=-2 k n$, Eq. (1) takes the standard form

$$
I(\omega)=S(\omega)\left|1+\int_{-\infty}^{+\infty} a(z) e^{-j \omega z} \mathrm{~d} z\right|^{2},
$$

which is the one that we shall consider. The FDOCT inverse problem can now be formulated explicitly as the task of computing $a(z)$ given $I(\omega)$ and $S(\omega)$.

\section{CONVENTIONAL FOURIER-BASED RECONSTRUCTION TECHNIQUE}

Developing the squares in Eq. (2), we obtain that

$$
\begin{aligned}
I(\omega)= & S(\omega)\left(1+\int_{-\infty}^{+\infty} a(z) e^{-j \omega z} \mathrm{~d} z+\int_{-\infty}^{+\infty} a^{*}(z) e^{j \omega z} \mathrm{~d} z\right. \\
& \left.+\int_{-\infty}^{+\infty} \int_{-\infty}^{+\infty} a(z) a^{*}\left(z^{\prime}\right) e^{-j \omega\left(z-z^{\prime}\right)} \mathrm{d} z \mathrm{~d} z^{\prime}\right) .
\end{aligned}
$$

We identify the following terms in Eq. (3).

- $\int_{-\infty}^{+\infty} a(z) e^{-j \omega z} \mathrm{~d} z$, also known as the Müller fringe term;

- $\int_{-\infty}^{+\infty} a^{*}(z) e^{j \omega z} \mathrm{~d} z$, the conjugate of the Müller fringe term; and

- $\int_{-\infty}^{+\infty} \int_{-\infty}^{+\infty} a(z) a^{*}\left(z^{\prime}\right) e^{-j \omega\left(z-z^{\prime}\right)} \mathrm{d} z \mathrm{~d} z^{\prime}$, which is the Fourier transform of the autocorrelation $r_{a a}(z)$, accounts for the mutual interference of all elementary waves.

Typically, $a(z)$ is one-sided; i.e., $a(z)=0$ for $z \leq z_{0}$. Under the assumption that the zero-delay plane is located outside the object, we have that $z_{0}>0$. In our subsequent developments, without loss of generality, we assume that $a(z)$ is real.

The standard reconstruction approach is to compute the inverse Fourier transform of $I(\omega)$. The tomogram in this case comprises $a(z), a(-z)$, and $r_{a a}(z)$. There is no overlap between $a(z)$ and $a(-z)$ because we assumed that $z_{0}>0 . r_{a a}(z)$ is symmetric about $z=0$ and overlaps with $a(z)$ and $a(-z)$. The overlap can be reduced-although not fully eliminated-by increasing $z_{0}$. However, this would cause the interference fringes in the power spectrum to move closer, requiring a higher-resolution spectrometer. This would also reduce the accessible depth, and the SNR, for a given spectrometer resolution. The best resolution, SNR, and maximal access in the axial direction are obtained for $z_{0}=0$. 


\section{EXACT RECONSTRUCTION}

Let $A(\omega)=\int_{-\infty}^{+\infty} a(z) e^{-j \omega z} \mathrm{~d} z$, where $a(z) \in\left(L^{1} \cap L^{2}\right)(\mathrm{R})$. Rewriting Eq. (2), we have that

$$
I(\omega)=S(\omega)(1+A(\omega))\left(1+A^{*}(\omega)\right) .
$$

The autocorrelation term is a consequence of the multiplicative interaction between $A(\omega)$ and $A^{*}(\omega)$. We propose to convert it to an additive one by considering the logarithm of $I(\omega)$. Thus, we have that

$$
\log I(\omega)-\log S(\omega)=\log (1+A(\omega))+\log \left(1+A^{*}(\omega)\right) .
$$

The right-hand side of Eq. (5) has some nice properties that enable exact recovery of $a(z)$ from $|1+A(\omega)|^{2}$. This key result is presented in the form of the following theorem:

Theorem 1. If $a(z) \in\left(L^{1} \cap L^{2}\right)(\mathbb{R})$ vanishes for $z<0 \leq z_{0}$ $\mathcal{F}$ and $a(z) \leftrightarrow A(\omega)$ such that $|A(\omega)| \leq \epsilon<1 \forall \omega$, then $\mid 1$ $+\left.A(\omega)\right|^{2}$ completely specifies a $(z)$ almost everywhere (a.e.).

Proof. Our proof is constructive. First, we need the following lemmas.

Lemma 1. If $a(z) \in\left(L^{1} \cap L^{2}\right)(\mathbb{R})$ vanishes for $z<0 \leq z_{0}$ $\mathcal{F}$

and $a(z) \leftrightarrow A(\omega)$ such that $|A(\omega)| \leq \epsilon<1 \forall \omega$, then the inverse Fourier transform of $\log (1+A(\omega))$ vanishes over $z$ $<0 \leq z_{0}$ almost everywhere.

Proof. By employing a Taylor series expansion, we have that

$$
\log (1+A(\omega))=\sum_{n=1}^{\infty}(-1)^{n-1} \frac{A^{n}(\omega)}{n} \text { for }|A(\omega)|<1, \forall \omega .
$$

Since $|A(\omega)| \leq \epsilon<1 \forall \omega, \log (1+A(\omega))$ is bounded and welldefined. The Fourier transform converts convolutions to multiplications so that

$$
(\underbrace{a * a * \cdots * a}_{n \text { times }})(z) \stackrel{\mathcal{F}}{\leftrightarrow} A^{n}(\omega)
$$

Since $a(z)$ vanishes for $z<0 \leq z_{0}$, the left-hand side of Eq. (7) also vanishes for $z<0 \leq z_{0}$, almost everywhere.

Similarly, it can be shown that the inverse Fourier transform of $\log \left(1+A^{*}(\omega)\right)$ vanishes for $z>z_{0} \geq 0$ almost everywhere.

Lemma 2. If $a(z) \in\left(L^{1} \cap L^{2}\right)(\mathbb{R})$ vanishes for $z \geq z_{0} \geq 0$ $\mathcal{F}$

and $a(z) \leftrightarrow A(\omega)$ such that $|A(\omega)| \leq \epsilon<1 \forall \omega$, then the inverse Fourier transform of $\log \left(1+A^{*}(\omega)\right)$ vanishes over $z$ $\geq z_{0} \geq 0$ almost everywhere.

Proof. The proof is similar to that of Lemma 1.

Having established lemmas 1 and 2, we continue with the proof. We know that

$$
\log |1+A(\omega)|^{2}=\log (1+A(\omega))+\log \left(1+A^{*}(\omega)\right) .
$$

Applying the inverse Fourier transform on both sides, we have that

$$
c(z)=\mathcal{F}^{-1}\left\{\log |1+A|^{2}\right\}(z)=\mathcal{F}^{-1}\left\{\log (1+A)+\log \left(1+A^{*}\right)\right\}(z) .
$$

The functions $\mathcal{F}^{-1}\{\log (1+A)\}(z)$ and $\mathcal{F}^{-1}\left\{\log \left(1+A^{*}\right)\right\}(z)$ have nonoverlapping support (by applying lemmas 1 and 2 ). This property is illustrated in Fig. 2. Now, define

$$
w(z)=\left\{\begin{array}{cc}
0, & z<-z_{0} \\
\frac{z}{2 z_{0}}+\frac{1}{2}, & -z_{0} \leq z \leq z_{0} . \\
1, & z>z_{0}
\end{array}\right.
$$

We therefore have that

$$
\mathcal{F}^{-1}\{\log (1+A)\}(z)=\mathcal{F}^{-1}\left\{\log |1+A|^{2}\right\}(z) w(z) \quad \text { a.e. }
$$

Applying the Fourier transform on both sides, we have that

$$
\begin{aligned}
\log (1+A(\omega)) & =\mathcal{F}\left\{\mathcal{F}^{-1}\left\{\log |1+A|^{2}\right\} w\right\}(z) \\
\Rightarrow A(\omega) & =\exp \left(\mathcal{F}\left\{\mathcal{F}^{-1}\left\{\log |1+A|^{2}\right\}(z) w\right\}(\omega)\right)-1
\end{aligned}
$$

so that $a(z)$ can be computed as $a(z)=\mathcal{F}^{-1}\{A\}(z)$ a.e.

\section{Remarks}

1. Theorem 1 is also applicable in the anticausal case where $a(z)$ vanishes over $z>z_{0}, z_{0} \leq 0$, provided that $w(z)$ in Eq. (10) is replaced by $w(-z)$. However, it is not applicable if $a(z)$ vanishes over $z<z_{0} \leq 0$.

2. From Eq. (6), note that, as $|\omega| \rightarrow+\infty, \log (1+A(\omega))$ decays as $A(\omega)$ and the higher-order terms $A^{n}(\omega)$ decay faster than $A(\omega)$. Therefore, the inverse Fourier transform of $\log (1+A(\omega))$ is well-defined in the classical sense. A similar result holds for $\log \left(1+A^{*}(\omega)\right)$.

3. The definition of $w(z), z \in\left[-z_{0}, z_{0}\right]$ is arbitrary since both $a(z)$ and $a(-z)$ vanish over that region, but a smooth $w(z)$ is preferred as it avoids ringing artifacts in practical implementations.

In some cases, it is of interest to recover complex $a(z)$ because it may be useful in characterizing the absorption properties of the specimen. To illustrate that Theorem 1 is applicable in this case as well, we consider $a(z)$ shown in Figs. 3(a) and 3(d). In Figs. 3(c) and 3(f), we show that $a(z)$ is exactly recovered by applying Theorem 1 . Comparing this with the conventional Fourier-based reconstruc-

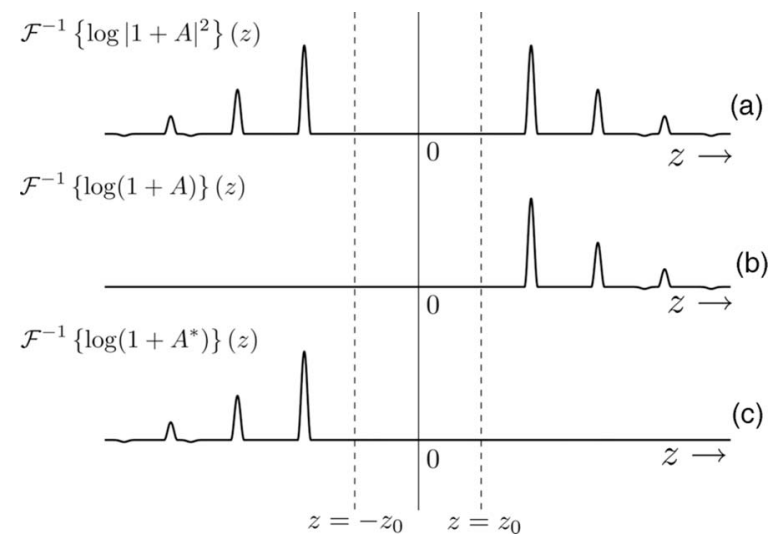

Fig. 2. Figure to illustrate the property that $\mathcal{F}^{-1}\{\log (1+A)\}(z)$ and $\mathcal{F}^{-1}\left\{\log \left(1+A^{*}\right)\right\}(z)$ have nonoverlapping support. 


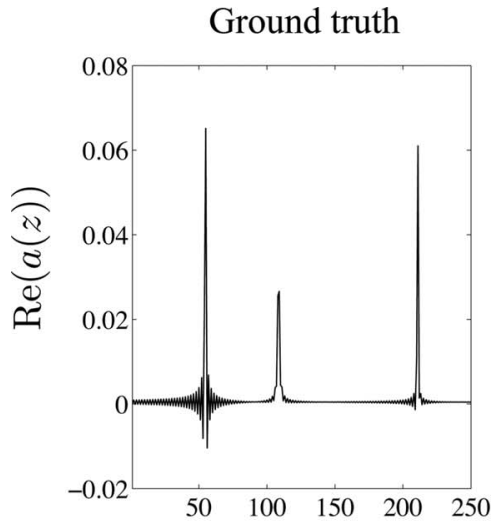

(a)

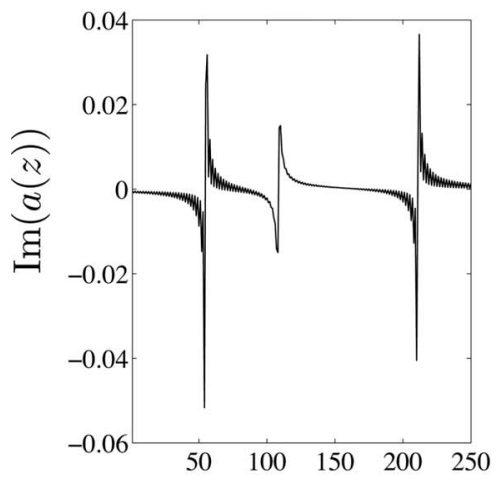

(d)
Fourier technique

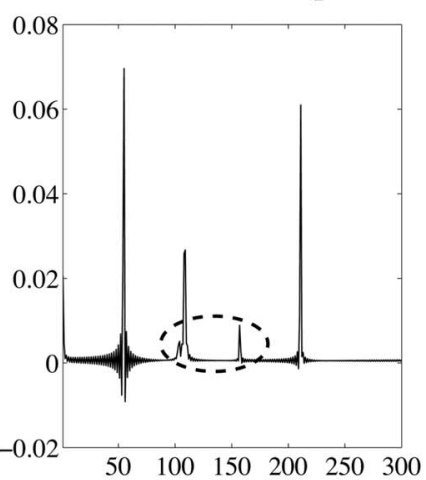

(b)

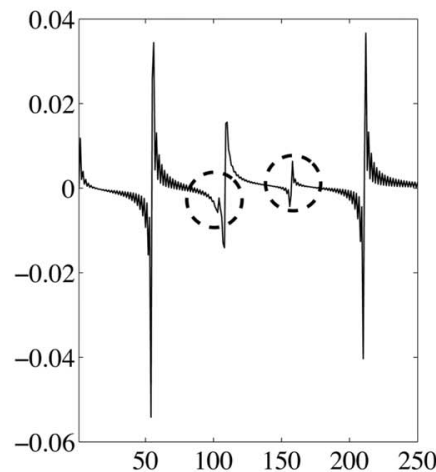

(e)
New technique

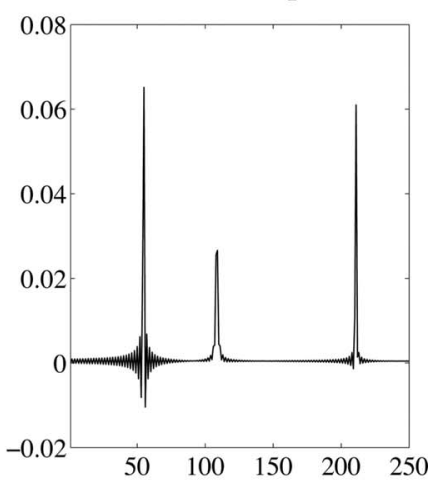

(c)

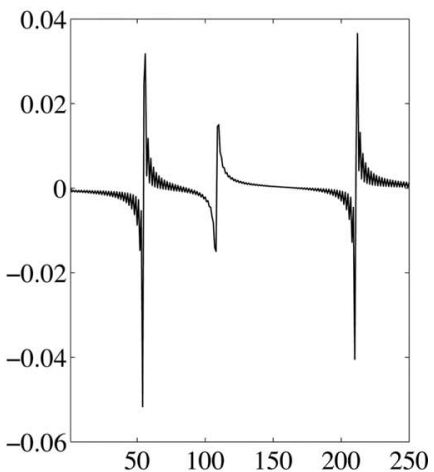

(f)

Fig. 3. Complex-function retrieval by application of Theorem 1. (a) and (d) are the real and imaginary parts of $a(z)$ (ground truth); (b) and (e) are the estimates obtained by using the conventional Fourier-transform technique (note the autocorrelation artifacts in the dashed circles); (c) and (f) are the estimates obtained by applying Theorem 1.

tions shown in Figs. 3(b) and 3(e), we note that the autocorrelation artifacts are suppressed to a large extent.

\section{EXACT RECOVERY AND HOMOMORPHIC SIGNAL PROCESSING}

The proof of Theorem 1 implicitly involves operators that are nonlinear in the conventional sense, but which satisfy a generalized principle of superposition. To explain further, we first provide a few definitions related to the cepstrum. For a detailed treatment of cepstrum see [20]. Our formulation is in the continuous-space and frequency domains, whereas the often-used one is a discrete-space, discrete-continuous-frequency domain formulation.

\section{A. Definition of Cepstrum}

Let $f(z) \in L^{2}(\mathbb{R})$ and $f(z) \leftrightarrow F(\omega)$. The complex cepstrum of $f(z)$ is defined as

$$
\hat{c}_{f}(z)=\frac{1}{2 \pi} \int_{-\infty}^{+\infty} \log F(\omega) e^{j \omega z} \mathrm{~d} \omega .
$$

$\hat{c}_{f}(z)$ exists and has finite energy if $\log F(\omega) \in L^{2}(\mathbb{R})$. The real cepstrum is defined as the inverse Fourier transform of $\log |F(\omega)|$; i.e.,

$$
c_{f}(z)=\frac{1}{2 \pi} \int_{-\infty}^{+\infty} \log |F(\omega)| e^{j \omega z} \mathrm{~d} \omega .
$$

From Eqs. (14) and (15), the following property can be verified:

$$
c_{f}(z)=\frac{\hat{c}_{f}(z)+\hat{c}_{f}^{*}(-z)}{2} .
$$

\section{B. Homomorphic Operators}

Let $T$ be an operator/system that maps $f_{1}(z)$ and $f_{2}(z)$ into $T\left\{f_{1}\right\}(z)$ and $T\left\{f_{2}\right\}(z)$, respectively. $T$ is said to satisfy the generalized principle of superposition if it satisfies the following properties under a given set of bivariate operations $\{\odot, \otimes, \sqcup, \triangle\}$ :

$$
T\left\{f_{1} \odot f_{2}\right\}(z)=\left\{T\left\{f_{1}\right\} \otimes T\left\{f_{2}\right\}\right\}(z) \text { (linearity), }
$$

$$
T\left\{c \sqcup f_{1}\right\}(z)=\left\{c \triangle T\left\{f_{1}\right\}\right\}(z) \text { (scaling property), }
$$




$$
f(z) \stackrel{\odot}{\longrightarrow} T\{., .\} \stackrel{\otimes}{\longrightarrow} g(z)
$$

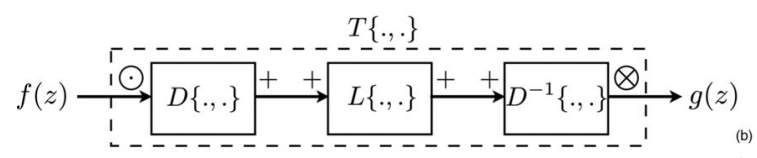

Fig. 4. (a) A homomorphic system and (b) its canonical representation.

where $c$ is a scalar. Such a system is said to be homomorphic with respect to the input and output operations $\odot$ and $\otimes$, respectively, and is denoted by $T\{\odot, \otimes\}$. A homomorphic system $T\{\odot, \otimes\}$ is equivalent to a cascade of three homomorphic systems [20]: $D\{\odot,+\}, L\{+,+\}$, and $D^{-1}\{+, \otimes\}$ (see Fig. 4), where the operator $D\{\odot,+\}$ is referred to as the characteristic system associated with the input operation $\odot, D^{-1}\{+, \otimes\}$ is the inverse characteristic system associated with the output operation $\otimes$, and where $L\{+,+\}$ is a linear system in the usual sense. The characteristic system and its inverse for the special case of $\odot$ $\equiv \otimes \equiv *$ are shown in Fig. 5 .

\section{FDOCT and Homomorphic Equivalence}

To establish the stated equivalence, we summarize the technique embedded in Theorem 1 below:

1. Background subtraction in the logarithm domain: given $I(\omega)$ and $S(\omega)$, compute $\log I(\omega)-\log S(\omega)$.

2. Inverse Fourier transformation: $c(z)=\mathcal{F}^{-1}\{\log I$ $-\log S\}(z)$.

3. Selection of the causal component: $c^{+}(z)=c(z) w(z)$.

4. Fourier transformation: $C^{+}(\omega)=\mathcal{F}\left\{c^{+}\right\}(\omega)$.

5. Exponentiation: $A(\omega)=\exp \left(C^{+}(\omega)\right)-1$.

6. Inverse Fourier transformation: $a(z)=\mathcal{F}^{-1}\{A\}(z)$.

If we include the acquisition of $I(\omega)$ and $S(\omega)$ as the 0 th step, then the operations in steps 4-6 form the inverse characteristic system shown in Fig. 5(b), corresponding to the operations in steps $0-2$, which form the characteristic system shown in Fig. 5(a). The function $c(z)$ is the real cepstrum of $\mathcal{F}^{-1}\{I / S\}(\omega)$. The multiplication in step 3 is equivalent to $L\{+,+\}$ in Fig. 4 and is referred to as "liftering". The function $c^{+}(z)$ is the complex cepstrum of $\mathcal{F}^{-1}\{1+A\}(z)$ and is identical to the causal part of $c_{f}(z)$ in Eq. (16). Thus, our technique has a one-to-one equivalence with the homomorphic system $T\{*, *\}$. By this equivalence we have formally shown that the FDOCT reconstruction problem is essentially the same as deconvo-
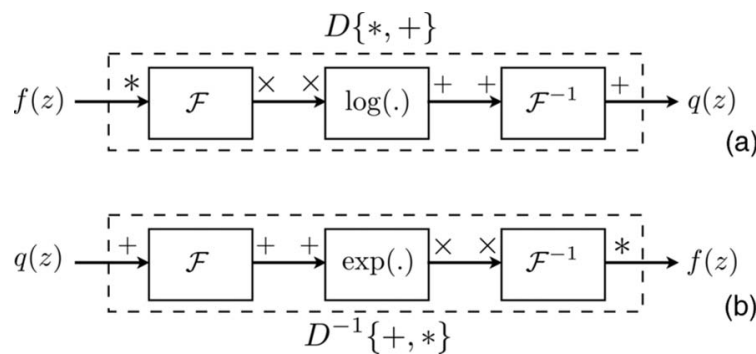

Fig. 5. (a) Characteristic system $D\{*,+\}$ for convolution and (b) its inverse $D^{-1}\{+, *\}$. lution. In the popular flavor of homomorphic deconvolution [20], the higher-order cepstral coefficients that depend on the measurements are separated from the lower-order ones coming mainly from the smoothing kernel. Deconvolution is achieved by retaining only the higher-order cepstral coefficients. In our technique, we have essentially performed deconvolution to separate $a(z)$ and $a(-z)$ by virtue of the property that their cepstra do not overlap.

\section{SAMPLING AND ALIASING ISSUES}

Let the discrete measurements along the wavelength axis be denoted by $\{I(m \Delta \lambda), m \in \mathbb{Z}\}$, where $\Delta \lambda$ is the sampling step. It is reasonable to assume that $a(z)$ has a compact support; i.e., $a(z)=0$ for $z \in \mathbb{R} \backslash\left[z_{0}, z_{0}+z_{\text {supp }}\right]$. For alias-free reconstruction of $I(\lambda)$ from $I(m \Delta \lambda), \Delta \lambda$ must satisfy the bandpass sampling theorem condition $\Delta \lambda \leq(B$ $+1) /\left[2\left(z_{0}+z_{\text {supp }}\right)\right]$, where $B$ is the largest integer such that $B \leq z_{0} / z_{\text {supp. }}$. Uniformly spaced measurements on the wavenumber axis can be obtained by a spline interpolation [23]. Let a set of $N$ such samples be denoted by $\{I(k), k=0,1,2, \ldots, N-1\}$. Consider the periodized version of $I(k)$ given by

$$
I_{p}(k)=\sum_{n=-\infty}^{+\infty} I(k+n N),
$$

which is the Fourier transform of the sampled spatialdomain function. This framework enables the use of discrete Fourier transform (DFT) for implementation.

The homomorphic technique involves the logarithm and exponential operations that can be a potential source of aliasing in finite length implementations. Aliasing can be reduced to an acceptable level by oversampling the measurements. The upsampler can be efficiently implemented by using a zero-padded fast Fourier transform (FFT) or by using $B$-splines [23]. In Fig. 6, we show the aliasing error in a synthesized scattering function for two cases: (i) no oversampling, and (ii) oversampling by a factor of 2. Oversampling by a mere factor of 2 suppresses the aliasing errors to an acceptable level. The amount of

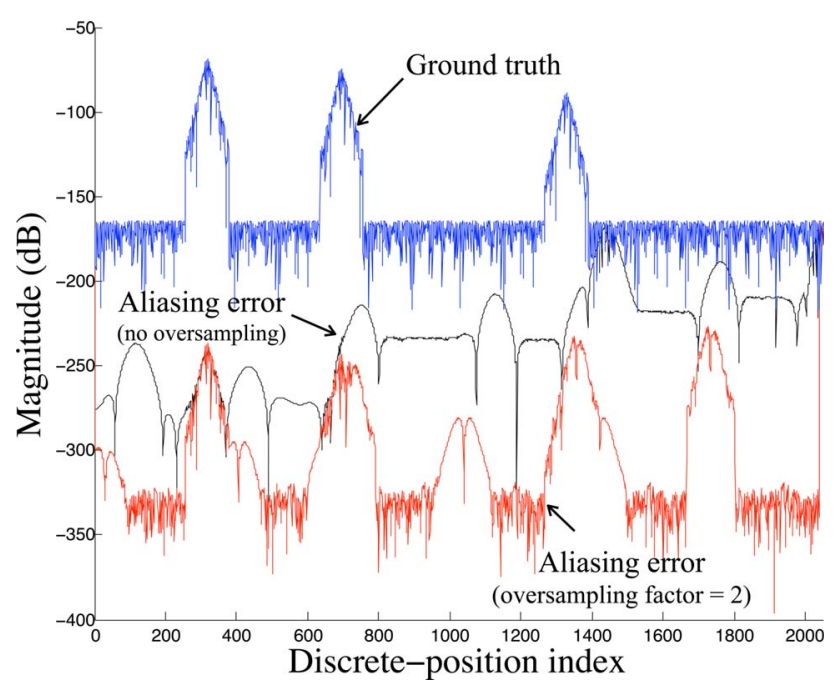

Fig. 6. (Color online) Spatial-domain aliasing as a function of the oversampling factor. 
aliasing is also a function of the ratio between the maximum of the scattering function and the source intensity. As $\max _{\omega}|A(\omega)|$ approaches unity, the aliasing errors increase for a fixed oversampling factor. Define the peak ratio as $\max _{\omega}|A(\omega)|: 1$. We conduct simulations to measure the aliasing error as a function of the oversampling factor and the peak ratio. To quantify the aliasing error, we define the signal-to-aliasing-error ratio as

$$
\eta=10 \log _{10}\left(\frac{\sum_{l=0}^{N-1} a^{2}(l \Delta z)}{\sum_{l=0}^{N-1}[a(l \Delta z)-\hat{a}(l \Delta z)]^{2}}\right),
$$

where $\Delta z$ is the sampling step, $a(l \Delta z)$ is the ground truth, and where $\hat{a}(l \Delta z)$ is an estimate of $a(l \Delta z)$. We show a plot of $\eta$ versus the oversampling factor as a function of the peak ratio in Fig. 7. If the peak ratio is less than $20 \%, \eta$ is sufficiently large and, therefore, no oversampling is necessary. For larger peak ratios, oversampling by a factor of 4 is necessary; beyond this factor, there is no appreciable improvement in $\eta$. In practice, the peak ratio is around $5 \%$ or less and upsampling by a factor of 2 seems to be sufficient. The associated computation cost comprises an $N$-point FFT and a $2 N$-point FFT after padding zeros.

In the above experiment we have not made any assumptions about the decay of $a(z)$. In practice, as $z$ increases, $a(z)$ decays at a rate determined by the specimen. If $a(z)$ decays quite fast, then the aliasing components will have little overlap with $a(z)$ and oversampling may not even be necessary.
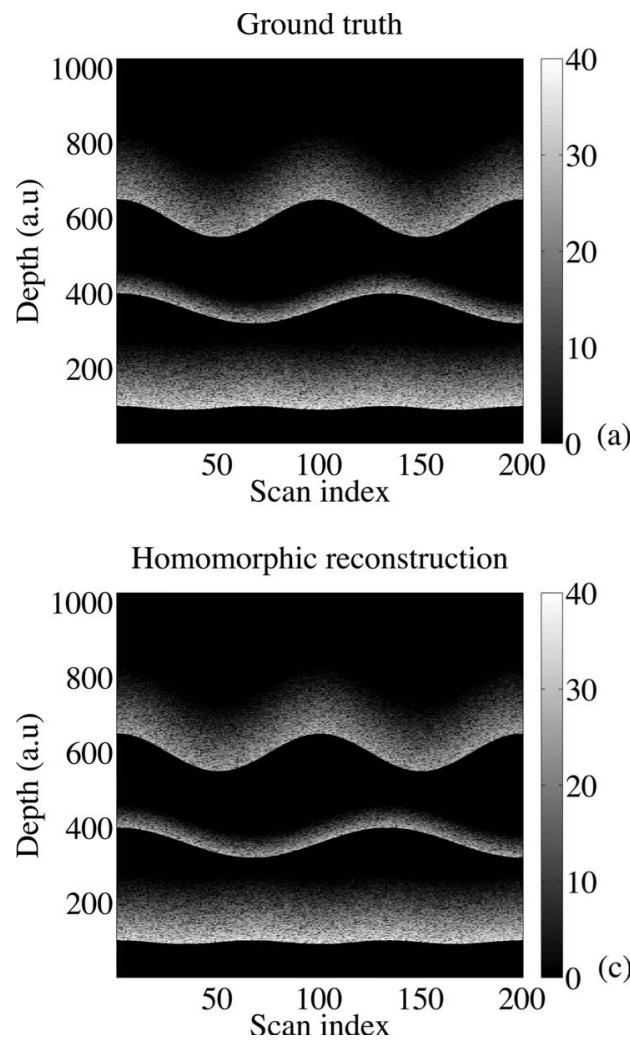

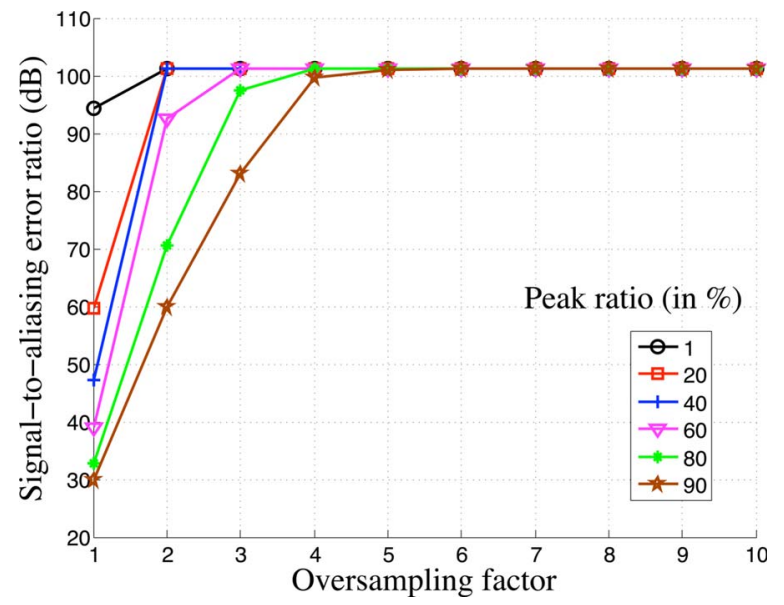

Fig. 7. (Color online) Signal-to-aliasing error ratio as a function of the oversampling factor.

\section{PERFORMANCE COMPARISON}

In this section, we compare the performances of the three techniques - the standard Fourier, the iterative [19], and the homomorphic-on synthesized and experimental data.

\section{A. Synthesized-Data Performance}

In Fig. 8, we show a synthesized multilayer specimen and the tomograms reconstructed by the three techniques mentioned above. The autocorrelation artifacts are prominent in the standard Fourier reconstruction whereas the homomorphic technique yields artifact-free reconstruction. The iterative technique gave nearly identical results
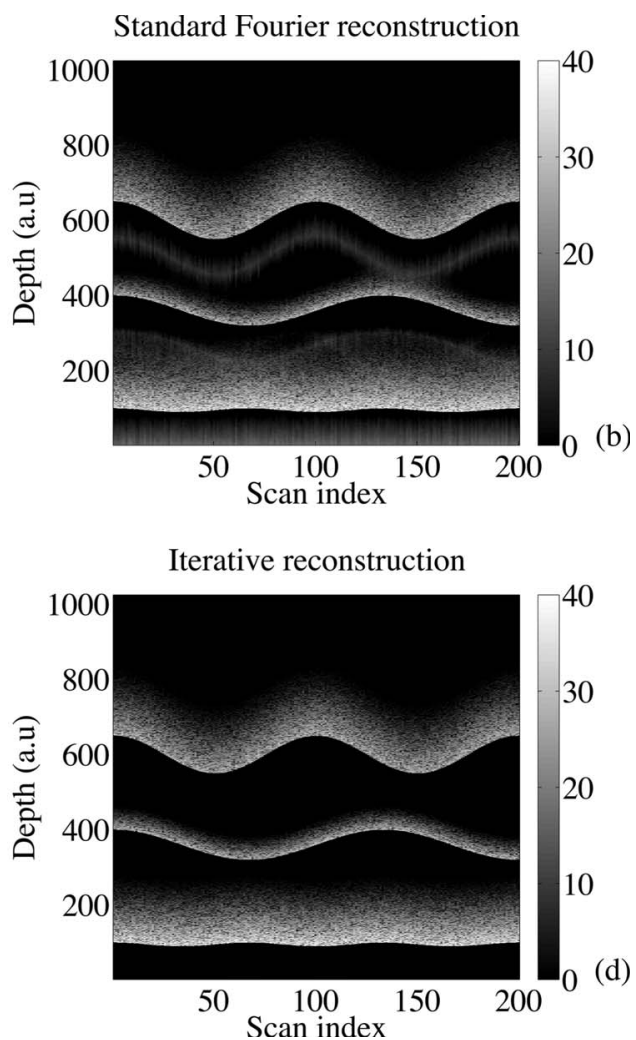

Fig. 8. Tomograms of a synthesized multilayer specimen: (a) ground truth, (b) standard Fourier reconstruction (notice the autocorrelation artifacts), (c) homomorphic reconstruction, and (d) iterative reconstruction. 
after 30 iterations. To quantify the artifact suppression, we introduce a new metric called the average signal-toartifact ratio,

$$
\xi=10 \log _{10}\left(\frac{1}{L} \sum_{l=1}^{L} \frac{\operatorname{Var}\left\{\mathbf{a}_{l}\right\}}{\operatorname{Var}\left\{\mathbf{a}_{l}-\hat{\mathbf{a}}_{l}\right\}}\right),
$$

where $L$ is the number of lateral scans; $\mathbf{a}_{l}$ $=\left\{a_{l}(i), i=1,2, \ldots, N\right\}, l=1,2, \ldots, L$, are the scattering functions corresponding to $L$ scans; $\hat{\mathbf{a}}_{l}$ is an estimate of $\mathbf{a}_{l}$; and where $\operatorname{Var}\{\cdot\}$ is the sample variance operator, acting on the elements of its vector argument. The value of $\xi$ is $25.34 \mathrm{~dB}$ for the conventional Fourier technique, $117.7 \mathrm{~dB}$ for the iterative technique, and $102.5 \mathrm{~dB}$ for the homomorphic technique. The processing times (in MATLAB) are $0.16,18.84$, and $1.52 \mathrm{~s}$. Recall that, in the absence of measurement noise, the accuracy of the homomorphic technique is limited only by aliasing. From these results we conclude that the homomorphic technique gives the
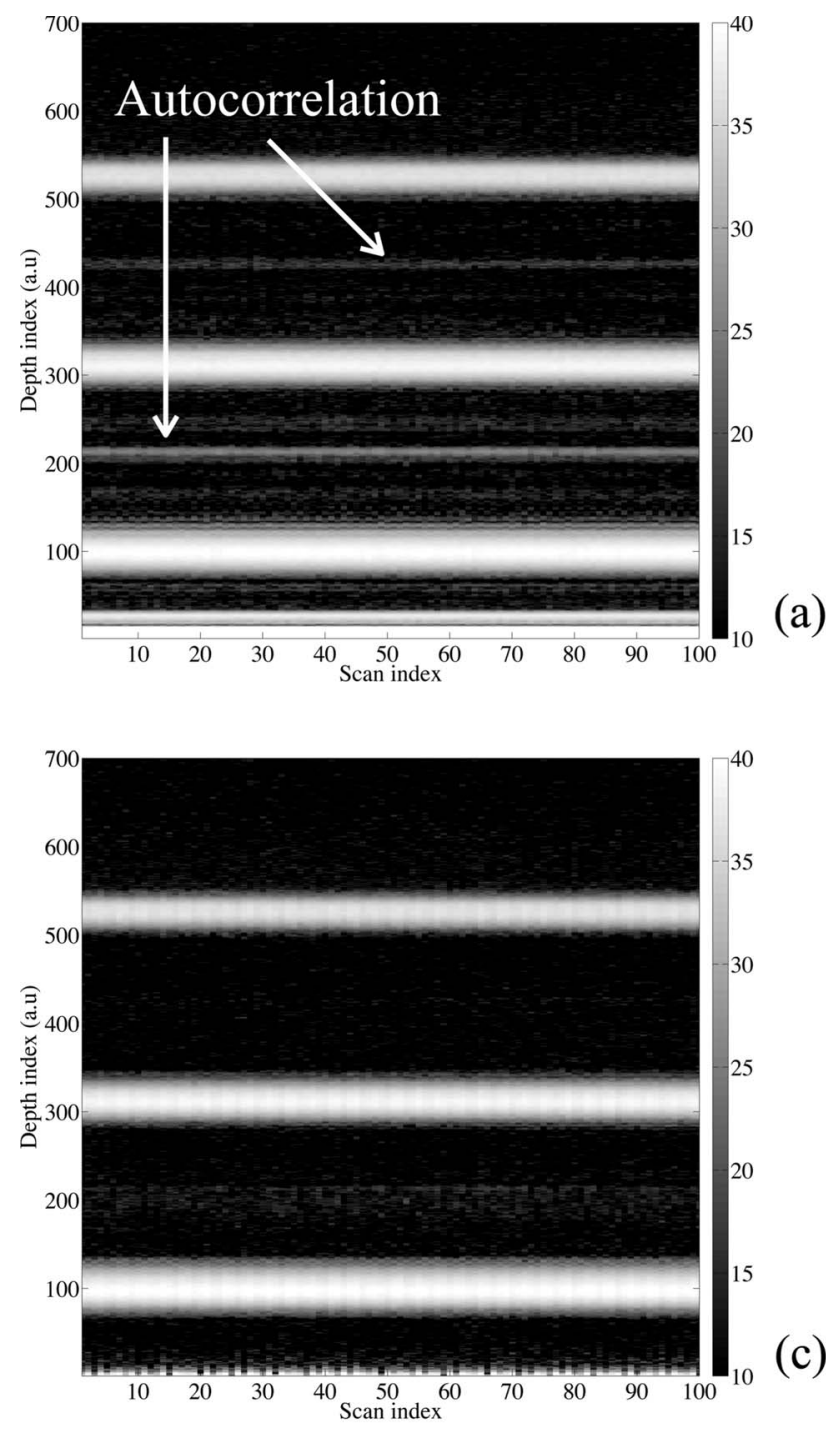

Fig. 9. Tomograms of a two-layered glass specimen obtained by the (a) conventional Fourier, (b) iterative, and (c) homomorphic techniques. The subplot (d) shows the standard deviation along scans as a function of depth for the three techniques. By comparing the standard deviation profiles in the dashed boxes, we note that the autocorrelation is significantly suppressed by the iterative and homomorphic techniques. The iterative technique has a performance that is superior to that of the conventional technique, but somewhat inferior to that of the homomorphic technique. best trade-off between computational efficiency and reconstruction accuracy.

\section{B. Computational Complexity}

The computational load of the standard technique is the least-one $N$-point FFT per scan, where $N$ is the sequence of measured spectral values. In the homomorphic technique, the computations comprise two $N$-point FFTs for upsampling, $2 \times 2 N$ logarithms, $2 N$ additions, a $2 N$-point inverse FFT, a $2 N$-point FFT of which $N$ elements are zeros, $2 N$ exponential operations, and a $2 N$-point inverse FFT. Thus, the total computation per scan comprises three $N$-point FFTs, $4 N$ logarithms, $2 N$ exponential operations, and $2 N$ additions. The computational load of the iterative technique is the highest. It comprises a $N$-point FFT, $N$-point FFT of a sequence which contains $N / 2$ consecutive zeros, $N$ arc-tangent operations, and $N$ complex multiplications. The computational load is further scaled

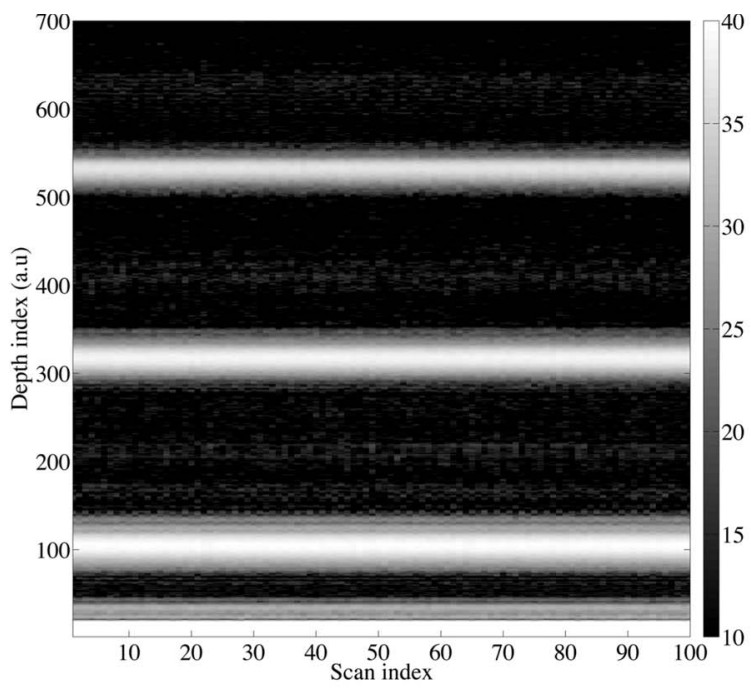

(b)

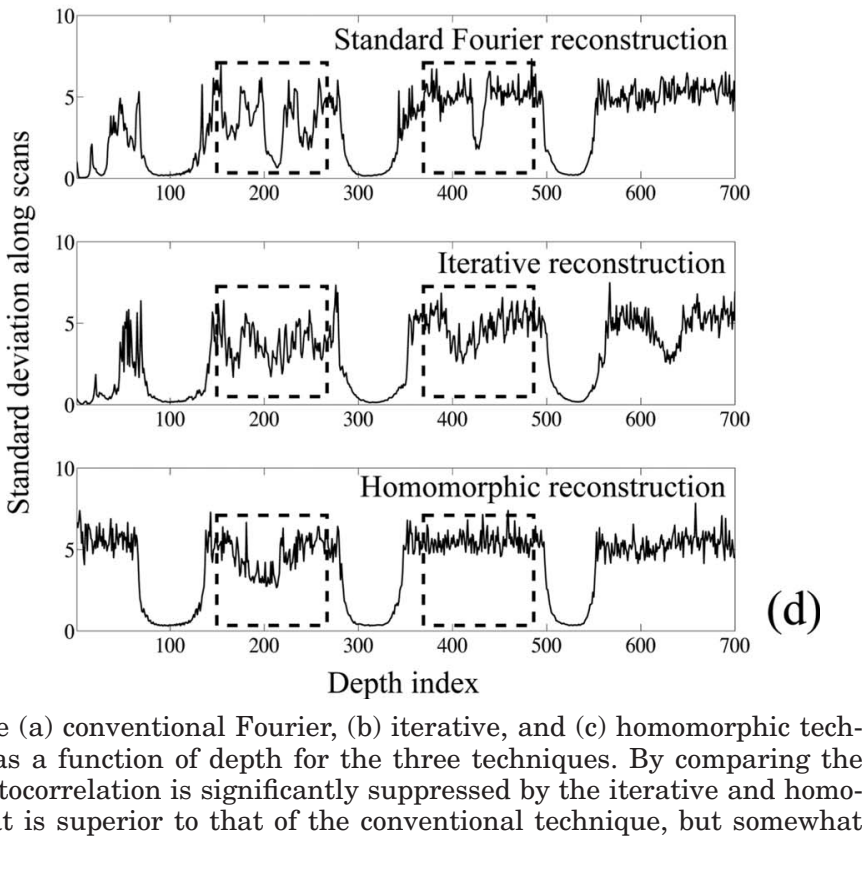


by the number of iterations. Thus, the homomorphic technique offers the best trade-off between accuracy and computational load.

\section{Experimental-Data Performance}

The experimental setup is the same as the one used for extended depth-of-focus applications [24]. The light source is a Ti-Sapphire laser with $\lambda_{0}=800 \mathrm{~nm}$ and $\Delta \lambda$ $=135 \mathrm{~nm}$. The beam is collimated by a lens having a focal length of $8.2 \mathrm{~mm}$ and split in reference and sample arms. In the sample arm, the beam passes through an axicon $\left(B=2.5^{\circ}\right)$. It is then focused by an achromatic lens system having a focal length of $177 \mathrm{~mm}$ into a thin annulus in the focal plane of the sample objective $(10 \times$ Zeiss Neofluor, numerical aperture $=0.3$ ). An identical objective is used for coupling the reference and scattered sample light into the detection fiber. The power at the sample is approximately $3 \mathrm{~mW}$. The axial resolution is $3 \mu \mathrm{m}$ in air and the lateral resolution is $1.3 \mu \mathrm{m}$. The integration time is $43 \mu \mathrm{s}$ and the line rate is $5 \mathrm{kHz}$.

We demonstrate our method on two specimens: (i) a two-layered glass sample and (ii) a mouse pancreas administered with $15 \%$ sucrose-such specimens are used in the study of diabetes. The measurements are processed by the three techniques, with some quality-enhancing modifications as described below. In the conventional technique, the Fourier transform is applied after subtracting the measured source spectrum. This operation, commonly known as background subtraction, is a standard preprocessing step and yields tomograms with higher contrast. In the iterative technique background subtraction cannot be done in the spectral domain because it destroys the minimum-phase property that is crucial for the technique. Therefore, we first reconstruct the tomogram without background subtraction. We process the source spectrum by the iterative technique. The minimum-phase source response thus obtained is subtracted from the reconstructed tomogram. In the homomorphic technique background subtraction is done in the log-spectral domain (step 1 of Subsection 5.C). Since this operation enhances the out-of-band measurement noise, we suppress it by convolving the reconstructed tomogram with the source response. Thus, in all three techniques the smoothing effect of the source on the tomogram is preserved.

In Fig. 9, we show the tomograms of the two-layered glass specimen obtained by the three techniques. The iterative technique converged in about 30 iterations. The autocorrelation introduced by the Fourier transform is clearly visible in Fig. 9(a); it is suppressed by the iterative

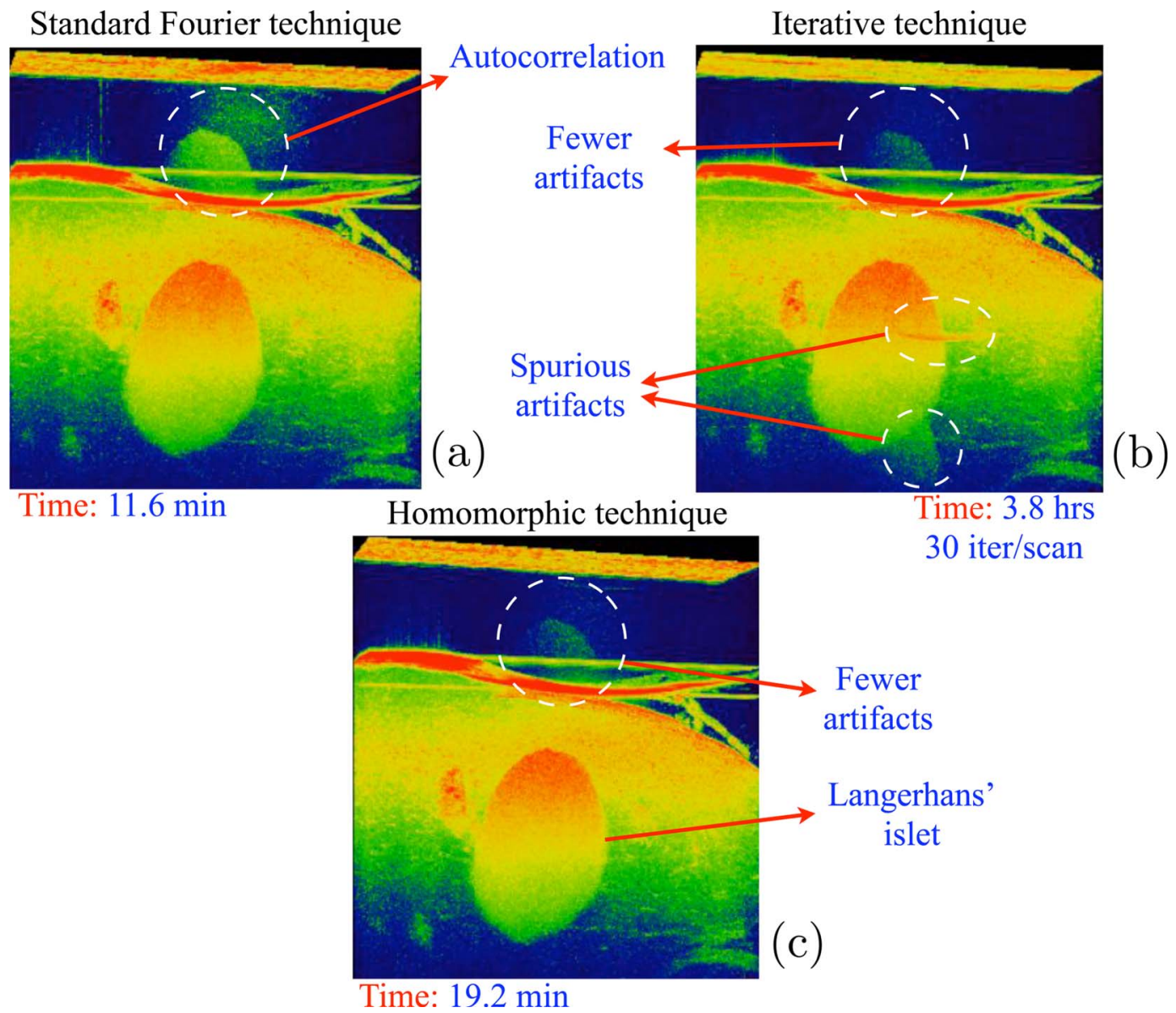

Fig. 10. (Color online) 3D visualization of a segment of a mouse pancreas: (a) standard Fourier, (b) iterative, and (c) homomorphic reconstructions. The $x$ step is $0.8 \mu \mathrm{m}$ and the $y$ step is $1.5 \mu \mathrm{m}$. The number of scans in the $x$ and $y$ directions is 1500 and 150 , respectively. The horizontal plane at the top corresponds to the zero-delay reference. The two horizontal lines are probably due to inaccuracies in estimating the background by averaging the scans. The tomogram reconstruction times, excluding the file access operations, are also indicated. The simulations are carried out in MATLAB 7.4 on a Macintosh 2.66 GHz dual-core Intel Xeon system. 
and homomorphic techniques [cf. Figs. 9(b) and 9(c), respectively]. Since this particular specimen has horizontal layers, an empirical way to further compare the reconstruction quality of the three techniques is to compute the standard deviation as a function of the depth index; the profiles thus obtained are shown in Fig. 9(d). We observe that the homomorphic technique has efficiently suppressed the autocorrelation terms to the level of the measurement noise. The performance of the iterative technique is superior to that of the standard Fourier technique but is somewhat inferior to that of the homomorphic technique.

In Fig. 10, we show the three-dimensional tomograms of a thin slice of a mouse pancreas containing some Langerhans' islets. The islets are cell clusters and constitute the endocrine part of the pancreas. The volumes shown are of size $1500 \times 150 \times 1024$, and they are rendered using the OsiriX medical image processing software [25]. The tomogram reconstruction times are also indicated. The intense reflection due to the moisture on the specimen surface gives rise to a strong autocorrelation in the conventional reconstruction [cf. Fig. 10(a)]; it is suppressed to a large extent by the iterative and homomorphic techniques [cf. Figs. 10(b) and 10(c), respectively]. Some distortions are still remaining and this may be due to a model mismatch, possibly caused by detection nonlinearities and/or measurement noise. Let us next compare Figs. 10(b) and 10(c). The iterative technique introduces some spurious distortions, which is not the case with the homomorphic technique. In terms of processing times, the homomorphic technique is much faster than the iterative technique. It takes only a few minutes (in MATLAB) to process a few hundred scans whereas the iterative technique takes a few hours. Thus, our technique provides a good trade-off between the quality of reconstruction and computational complexity.

\section{CONCLUSIONS}

We showed that exact signal reconstruction is possible from frequency-domain measurements. Our technique is noniterative, nonlinear, and offers an exact solution in the absence of noise, provided that the zero-delay plane is outside the specimen. We have also established an equivalence with homomorphic signal processing. By means of performance analysis on synthesized and experimental data, we have shown that our technique is superior to the iterative and conventional Fourier-transform techniques. Given the homomorphic reconstruction technique, the object can be placed very close to the zero-delay plane without introducing autocorrelation distortion. This configuration yields higher sensitivity and increases the accessible depth in the specimen. The homomorphic technique can also make a big difference in microscopy applications where the glass slide that is used to cover the specimen causes a strong autocorrelation. The potential nonbiological applications are in multilayer optical storage, document security, object identification, fault detection, etc. In these applications, full-field OCT [26] is used as it is more economical and enables faster imaging. The signal model is the same as that in Eq. (2) except that the functions are two-dimensional. It must be noted that since our technique requires a causal scattering function, it is not suitable for full-range reconstruction. Multidimensional extensions of the technique are also promising for digital holography $[27,28]$.

\section{ACKNOWLEDGMENTS}

This work has been supported in part by the Center for Biomedical Imaging (CIBM) of the Geneva-Lausanne universities and the EPFL, and the Hasler, Leenards, LouisJeantet, and Swiss National foundations. The authors thank Philippe Thévenaz for careful reading of the manuscript, Adrian Bachmann and Theo Lasser for fruitful discussions, and Jessica Dessimoz for preparing the biological samples.

\section{REFERENCES}

1. A. F. Fercher, C. K. Hitzenberger, G. Kamp, and S. Y. El-Zaiat, "Measurement of intraocular distances by backscattering spectral interferometry," Opt. Commun. 117, 43-48 (1995).

2. E. Wolf, "Three-dimensional structure determination of semi-transparent objects from holographic data," Opt. Commun. 1, 153-156 (1969).

3. G. Hausler and M. W. Lindner, "Coherence radar and spectral radar-new tools for dermatological analysis," J. Biomed. Opt. 3, 21-31 (1998).

4. N. A. Nassif, B. Cense, B. H. Park, M. C. Pierce, S. H. Yun, B. E. Bouma, G. J. Tearney, T. C. Chen, and J. F. de Boer, "In vivo high-resolution video-rate spectral-domain opticalcoherence tomography of the human retina and optic nerve," Opt. Express 12, 367-376 (2004).

5. M. Wojtkowski, T. Bajraszewski, I. Gorczynska, P. Targowski, A. Kowalczyk, W. Wasilewski, and C. Radzewicz, "Ophthalmic imaging by spectral opticalcoherence tomography," Am. J. Ophthalmol. 138, 412-419 (2004).

6. U. Schmidt-Erfurth, R. A. Leitgeb, S. Michels, B. Povazay, S. Sacu, B. Hermann, C. Ahlers, H. Sattmann, C. Scholda, A. F. Fercher, and W. Drexler, "Three-dimensional ultrahigh-resolution optical-coherence tomography of macular diseases," Invest. Ophthalmol. Visual Sci. 46, 3393-3402 (2005).

7. M. Wojtkowski, R. Leitgeb, A. Kowalczyk, T. Bajraszewski, and A. Fercher, "In vivo human retinal imaging by Fourierdomain optical-coherence tomography," J. Biomed. Opt. 7, 457-463 (2002).

8. A. F. Fercher, R. A. Leitgeb, C. K. Hitzenberger, H. Sattmann, and M. Wojtkowski, "Complex spectral interferometry OCT,” Proc. SPIE 3564, 173-178 (1999).

9. M. Wojtkowski, A. Kowalczyk, R. A. Leitgeb, and A. Fercher, "Full-range complex spectral optical-coherence tomography technique in eye imaging," Opt. Lett. 27, 1415-1417 (2002).

10. Y. Yasuno, S. Makita, T. Endo, G. Aoki, H. Sumimura, M. Itoh, and T. Yatagai, "One-shot-phase-shifting Fourierdomain optical-coherence tomography by reference wavefront tilting," Opt. Express 12, 6184-6191 (2004).

11. R. A. Leitgeb, C. K. Hitzenberger, A. F. Fercher, and T. Bajraszewski, "Phase-shifting algorithm to achieve highspeed long-depth-range probing by frequency-domain optical-coherence tomography,” Opt. Lett. 28, 2201-2203 (2003).

12. M. A. Choma, C. Yang, and J. A. Izatt, "Instantaneous quadrature low-coherence interferometry with $3 \times 3$ fiberoptic couplers," Opt. Lett. 28, 2162-2164 (2003).

13. A. H. Bachmann, R. A. Leitgeb, and T. Lasser, "Complex ultrahigh resolution Fourier-domain optical-coherence tomography," Proc. SPIE 6079, 60790 (2006).

14. A. Szkulmowska, M. Wojtkowski, I. Gorczynska, T. 
Bajraszewski, M. Szkulmowski, P. Targowski, A. Kowalczyk, and J. J. Kaluzny, "Coherent noise-free ophthalmic imaging by spectral optical coherence tomography," J. Phys. D 38, 2606-2611 (2005).

15. J. R. Fienup, "Phase retrieval algorithms: a comparison," Appl. Opt. 21, 2758-2769 (1982).

16. H. H. Bauschke, P. L. Combettes, and D. Luke, "Phase retrieval, error reduction algorithm, and Fienup variants: a view from convex optimization," J. Opt. Soc. Am. A 19, 1334-1345 (2002).

17. T. F. Quatieri, Jr. and A. V. Oppenheim, "Iterative techniques for minimum phase signal reconstruction from phase or magnitude," IEEE Trans. Acoust., Speech, Signal Process. 29, 1187-1193 (1981).

18. N. Nakajima, "Phase retrieval using the logarithmic Hilbert transform and the Fourier-series expansion," J. Opt. Soc. Am. A 5, 257-262 (1988).

19. A. Ozcan, M. J. F. Digonnet, and G. S. Kino, "Minimumphase-function-based processing in frequency-domain optical-coherence tomography systems," J. Opt. Soc. Am. A 23, 1669-1677 (2006).

20. A. V. Oppenheim and R. W. Schafer, Discrete-Time Signal Processing, 2nd ed. (Prentice-Hall, 1999).
21. D. G. Childers, D. P. Skinner, and R. C. Kemerait, "The cepstrum: a guide to processing," Proc. IEEE 65, 1428-1443 (1977).

22. B. E. Bouma and G. J. Tearney, eds., Handbook of Optical Coherence Tomography (Marcel Dekker, 2002).

23. M. Unser, "Splines: a perfect fit for signal and image processing," IEEE Signal Process. Mag. 16, 22-38 (1999)

24. R. A. Leitgeb, M. L. Villiger, A. H. Bachmann, L. Steinmann, and T. Lasser, "Extended focus depth for Fourier-domain optical-coherence tomography," Opt. Lett. 31, 2450-2452 (2006).

25. A. Rosset, J. Heuberger, and O. Ratib, "OsiriX imaging software" (2007), http://www.osirix-viewer.com/.

26. S. Chang, X. Cai, and C. Flueraru, "Image enhancement for multilayer information retrieval by using full-field opticalcoherence tomography," Appl. Opt. 45, 5967-5975 (2006).

27. E. Cuche, "Numerical reconstruction of digital holograms: application to phase-contrast imaging and microscopy," Ph.D. dissertation (Ecole polytechnique fédérale de Lausanne (EPFL), 2000).

28. M. Liebling, T. Blu, and M. Unser, "Complex-wave retrieval from a single off-axis hologram," J. Opt. Soc. Am. A 27, $367-377$ (2004). 